\title{
Simulated Tests of Feedforward Active Noise Control (ANC) for Building Noise Cancellation
}

\author{
Tongrui Peng ${ }^{1}$, Quanmin $\mathrm{Zhu}^{1 *}$, M.O. Tokhi ${ }^{2}$ and Yufeng Yao ${ }^{1}$ \\ ${ }^{1}$ Faculty of Environment and Technology- Engineering, Design and Mathematics, \\ University of the West of England, U.K \\ *Quan.Zhu@uwe.ac.uk \\ ${ }^{2}$ Department of the Electrical and Electronic Engineering Division within the School \\ of Engineering, London South Bank University (LSBU), U.K
}

\begin{abstract}
This student paper presents an understanding of Active Noise Control (ANC) systems especially focus on the physical geometry about the detector and the observer and develops a series of simulation experiments for the different type of signal to test the cancellation performance using feedforward active noise control procedure with the fixed controller proposed by Tokhi and Leitch in 1987. This study will provide a further demonstration for the control approach and a foundation for proposing new ANC algorithms in the future studies.
\end{abstract}

Keywords: Building Noise, Low-frequency Noise, Active Noise Control, Feedback ANC Control, Feedforward ANC Control, ANC with Fixed Controller.

\section{Introduction}

Building noise and many engineering processes are important aspects in today's daily life and it comes with its consequences in severely affecting people's living quality. This is both in physical and psychological aspects [1], especially with relation to both human comfort and indoor environmental quality [2], [3].

Active noise control is an advanced and efficient technology for low frequency (lower than $500 \mathrm{~Hz}$ ) unwanted sound cancellation. It should be noted that wavelengths at low frequencies become larger compared with conventional acoustic absorbers [4].

Lueg in America proposed the first concept for Active Noise Control (ANC) in 1936 and several literature reviews have summarized the development of ANC [4], [5], [6], [7]. The whole Active Noise Control (ANC) system contains three main parts based on the principle of superposition. The first part is detection with the aim of detecting a signal coherent with the unwanted (primary) noise. The microphone is used in this step as a detector sensor due to its own flat amplitude and nearly linear characteristics. After noise is detected by the microphone, it will be transformed into an electrical signal. The second part is negation. It is the most important and inevitable part of the whole ANC system as the role of the controller is to adjust both the amplitude and phase for each of 
the primary source components. The aim of this step is to ensure that the secondary source has the same amplitude as the reverse phase (180 degrees corresponding to the primary source). Cancellation is the final part of the process, otherwise known as superposition. Another electrical device loudspeaker is placed at a fixed distance away from the primary source to transfer the generated electrical signal into audio sound. The results of the superimposing waves can be observed by the observer microphone [5], [8], [9], [10].

In 1987, Tokhi and Leitch's published a paper using the mathematical expression to explore how to set the detector and observer to attend high attenuation for non-dispersive primary source in two or three-dimensional space [5]. Prior to this little was found about geometrical considerations in other literatures.

In this paper, several simulated experiments were executed using Matlab Simulink software to demonstrate the performance of the feedforward active noise control system. A fixed controller was used with the different value of distance ratios for noise cancellation. What's more, related discussions regarding how to select a certain type of fixed controller and how to set the observer and detector appropriately are provided.

\section{ANC Structure (Tokhi and Leitch, 1987)}

ANC structure depends on the location of the microphone, controller, loudspeaker, and due to there are lots of configuration of these transducers and devices, and there is a tremendous type of ANC structures. These structures can be classified into two basic types: Feedback ANC system and Feedforward ANC system.

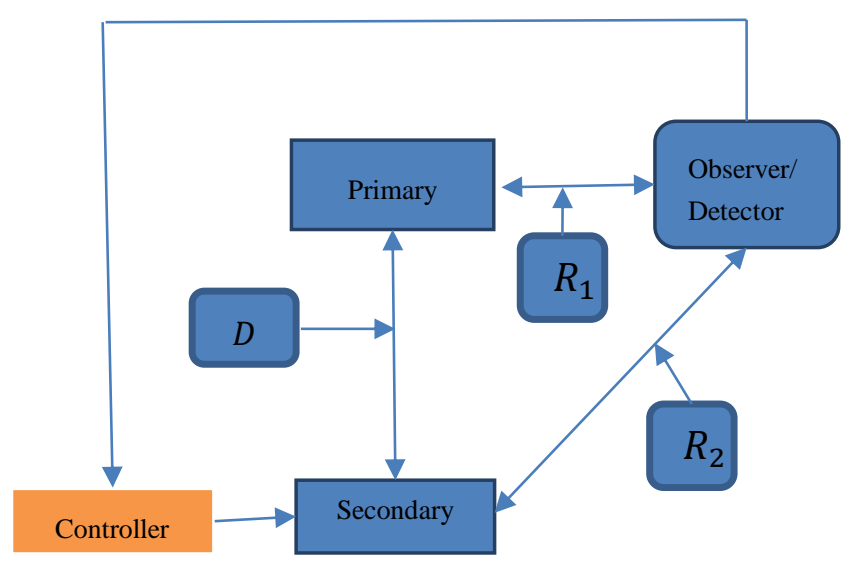

Fig. 1. Schematic diagram of Feedback ANC System [3]

$D$ : Physical distance between primary sources and secondary sources 
$R_{1}$ : Physical distance between primary sources to observer/detector

$R_{2}$ : Physical distance between secondary sources to observer/detector

Figure 1 is the geometry configuration about feedback ANC system from Tokhi and Leitch's publication in 1987. The primary source emits a pressure wave, which is detected by the detector, located at a distance of $R_{1}$ relative to the primary source. The microphone then transfers the acoustic wave to the electrical signal and then pass to the controller. The controller here is required to adjust the amplitude and phase of each component of the detected signal and later it will be emitted as secondary sources. The optimal situation is that at the observer point, zero sound pressure level (SPL) can be observed. The characteristics of feedback ANC system is that observer coincides with the detector.

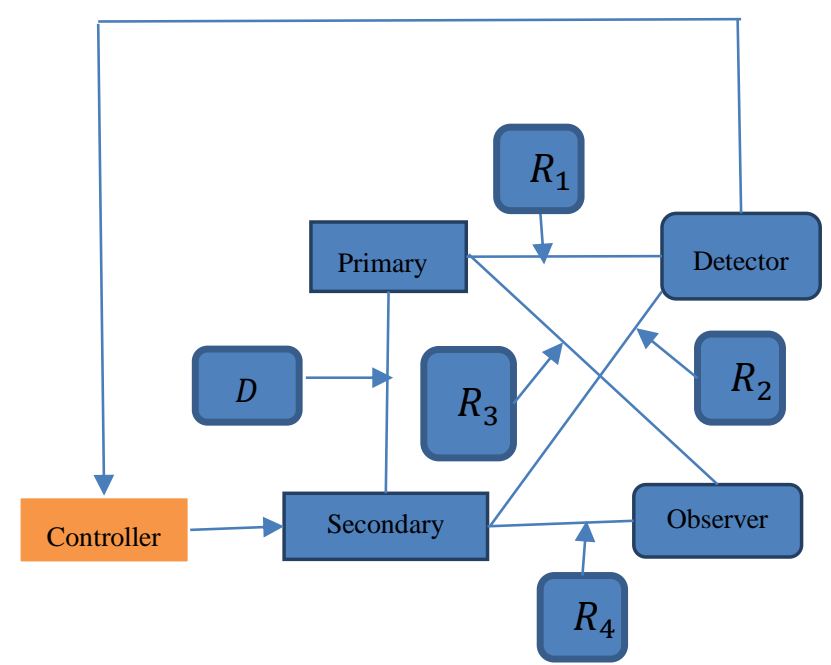

Fig. 2. Schematic diagram of Feedforward ANC System [3]

$D$ : Physical distance between primary sources and secondary sources

$R_{1}$ : Physical distance between primary sources to the detector

$R_{2}$ : Physical distance between secondary sources to the detector

$R_{3}$ : Physical distance between primary sources to the observer

$R_{4}$ : Physical distance between secondary sources to the observer

Figure 2 is the geometry configuration of feedforward active noise control (ANC) system from Tokhi and Leitch's publication in 1987. Compared with feedback ANC system, the detector and observer are placed at a separated place with different distance relative to the primary source and the secondary source. 


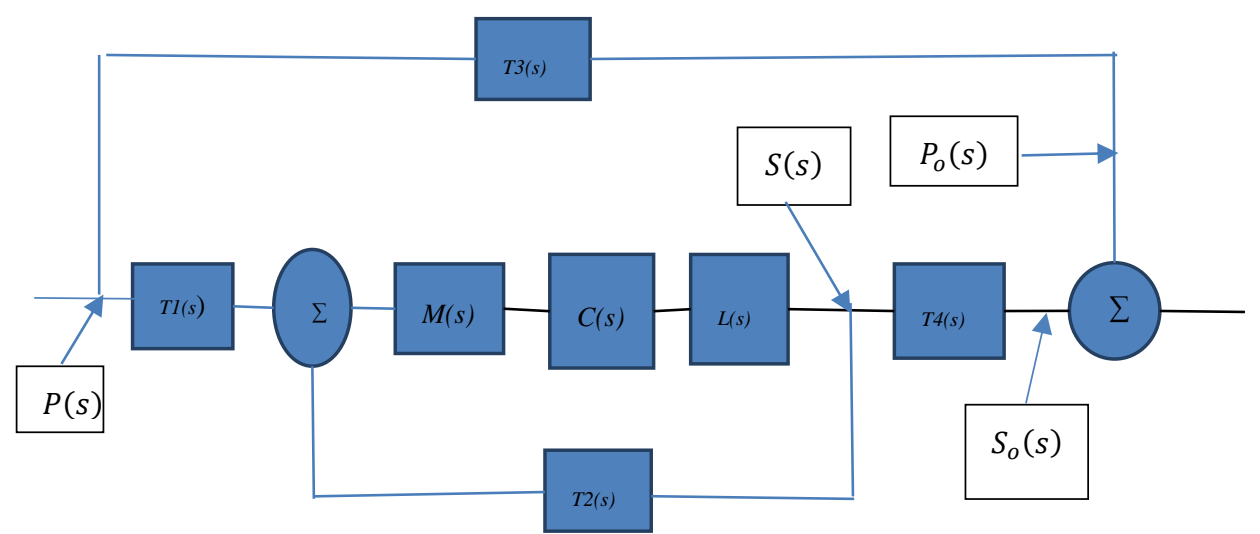

Fig. 3. Block diagram of Feedforward ANC System [3]

$T 1(s)$ : transfer characteristics of path $R_{1}$

$T 2(s)$ : transfer characteristics of path $R_{2}$

T3(s): transfer characteristics of path $R_{3}$

T4(s): transfer characteristics of path $R_{4}$

$M(s)$ : transfer characteristics of the microphone

$L(s)$ : transfer characteristics of the loudspeaker

$C(s)$ : transfer characteristics of the controller

From the figure 3 above, it is easier to get the primary source at the observation point.

$$
P_{o}(s)=T 3(s) P(s)
$$

The Secondary source at the observation point.

$$
S_{o}(s)=T 4(s) S(s)
$$

The mathematical expression for the secondary source at the loudspeaker.

$$
S(s)=[T 2(s) S(s)+T 1(s) P(s)] M(s) C(s) L(s)
$$

The expected results at the observation point.

$$
P_{o}(s)+S_{o}(s)=0
$$

Then the required controller continuous-frequency transfer function can be obtained.

$$
C(s)=\frac{T 3(s)}{M(s) L(s)[T 2(s) T 3(s)-T 1(s) T 4(s)]}
$$

Equation (5) is the required transfer function for optimum cancellation for feedforward active noise control system.

In figure 2, if detector or observer is located at equidistance relative to primary sources and secondary sources, the whole controller is called as fixed controller and equation (6) represents the controller's transfer function when the observer is located at equidistance from two sources.

$$
C(s)=\frac{1}{M(s) L(s)[T 2(s)-T 1(s)]}
$$


Theoretically, the optimal cancellation will occur if both transducers are located equidistance from the primary source and the secondary source or the distance ratio for both transducers relative to sound sources is equal. Under such condition, the controller requires for infinite larger gain and it is impossible to implement in fact.

\section{$3 \quad$ Simulation studies}

In this section, four simulated experiments will be executed on Matlab Simulink platform for two types of feedforward ANC system. The first type is that the detector is located equidistance relative to both sound sources and the second type is that the observer is located equidistance relative to both sound sources. Besides, additional exploration focus on whether the location of transducers will affect noise cancellation performance for feedforward ANC control with a fixed controller.

\subsection{Single Frequency Signal}

A sine wave $y=\sin (t)$ is used for primary (unwanted) source and the fundamental simulation time step is fixed while the total simulation time is 50 seconds.
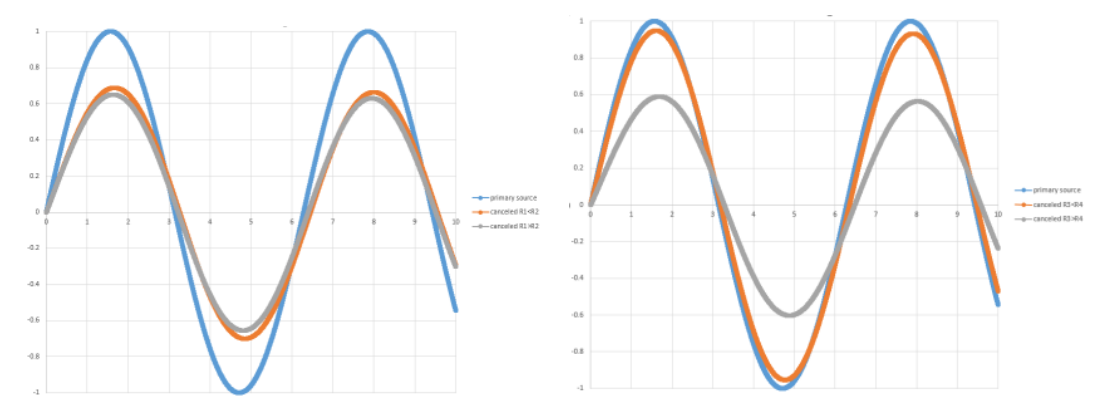

Fig. 4.1. Comparison between primary sources and canceled results $R_{3}=R_{4}, R_{1}<R_{2} \& R_{1}>$ $\mathrm{R}_{2}$ (left); $\mathrm{R}_{1}=\mathrm{R}_{2}, \mathrm{R}_{3}<\mathrm{R}_{4} \& \mathrm{R}_{3}>\mathrm{R}_{4}$ (right)

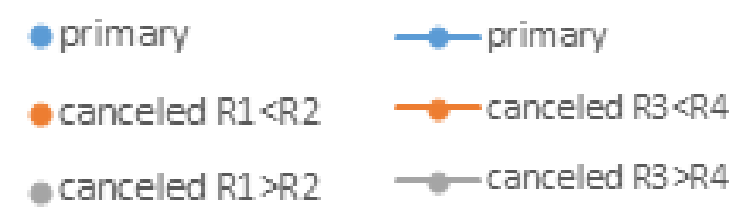

Fig. 4.2. Color explanation for simulation results: $R_{3}=R_{4}, R_{1}<R_{2} \& R_{1}>R_{2}$ (left); $R_{1}=$ $\mathrm{R}_{2}, \mathrm{R}_{3}<\mathrm{R}_{4} \& \mathrm{R}_{3}>\mathrm{R}_{4}$ (right) 
The $\mathrm{x}$-axis refers to time while the y-axis refers to the amplitude of the signal. For the single frequency signal, the system can achieve the aim of cancellation but the cancellation performance depends on the configuration of transducers. When $R_{3}=R_{4}$, the maximum extent of cancellation is nearly $36 \%$ while $R_{1}=R_{2}$, the maximum extent of cancellation can reach $40 \%$. The common performance is that for single frequency signal when the transducer is placed closer to secondary source than the primary source, the cancellation performance is better especially put the detector at an equidistance from two sound sources.

\subsection{Multiple-frequency signal}

For the multiple-frequency signal framework, the only difference compared with the first simulation test is that primary source changes to $y=\sin (t)+\sin (10 * t)$. The fundamental simulation time step and the whole simulation time keep the same.
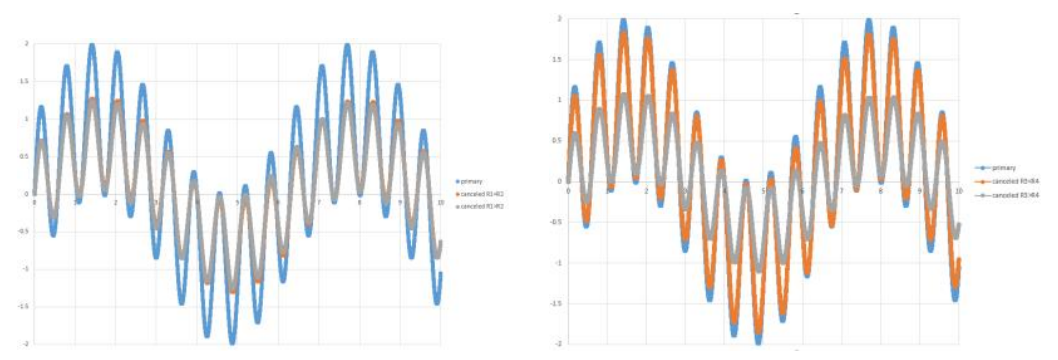

Fig. 5.1. Comparison between primary sources and canceled results $R_{3}=R_{4}, R_{1}<R_{2} \& R_{1}>$ $\mathrm{R}_{2}$ (left); $\mathrm{R}_{1}=\mathrm{R}_{2}, \mathrm{R}_{3}<\mathrm{R}_{4} \& \mathrm{R}_{3}>\mathrm{R}_{4}$ (right)

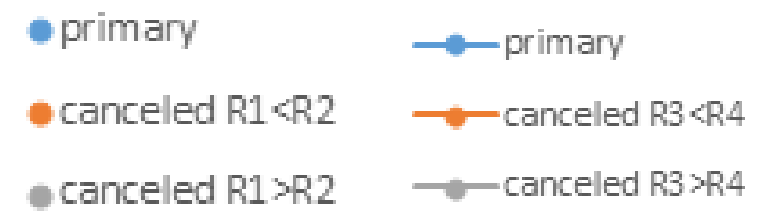

Fig. 5.2. Color explanation for simulation results: $R_{3}=R_{4}, R_{1}<R_{2} \& R_{1}>R_{2}$ (left); $R_{1}=$ $\mathrm{R}_{2}, \mathrm{R}_{3}<\mathrm{R}_{4} \& \mathrm{R}_{3}>\mathrm{R}_{4}$ (right)

The $\mathrm{x}$-axis refers to time while the y-axis refers to the amplitude of the signal. For the multiple-frequency signal, the system can achieve the aim of cancellation but the cancellation performance depends on the configuration of transducers. When $R_{3}=R_{4}$, the maximum extent of cancellation is nearly $37 \%$ while $R_{1}=R_{2}$, the maximum extent of cancellation can reach $38.9 \%$. Similar to the previous test, when the transducer is placed 
Simulated Tests of Feedforward Active Noise Control (ANC) for Building Noise Cancellation

closer to secondary source than the primary source, the cancellation performance is better especially put the detector at an equidistance from two sound sources.

\subsection{Multiple-frequency signal with Gaussian White Noise}

For this simulation case, add Gaussian white noise to multiple-frequency signaly $=$ $\sin (t)+\sin (10 * t)$. The fundamental simulation time step and the whole simulation time keep the same.
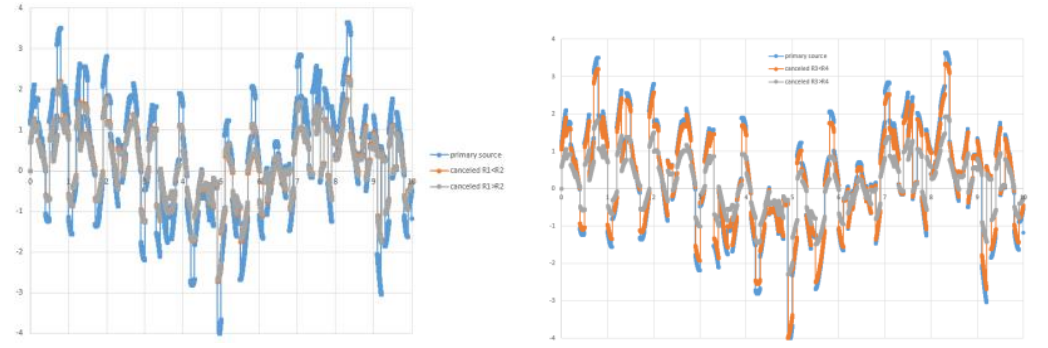

Fig. 6.1. Comparison between primary sources and canceled results: $R_{3}=R_{4}, R_{1}<$ $\mathrm{R}_{2} \& \mathrm{R}_{1}>\mathrm{R}_{2}$ (left); $\mathrm{R}_{1}=\mathrm{R}_{2}, \mathrm{R}_{3}<\mathrm{R}_{4} \& \mathrm{R}_{3}>\mathrm{R}_{4}$ (right)

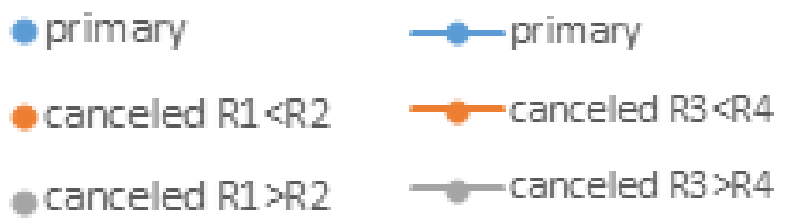

Fig. 6.2. Color explanation for simulation results: $R_{3}=R_{4}, R_{1}<R_{2} \& R_{1}>R_{2}$ (left); $R_{1}=$ $\mathrm{R}_{2}, \mathrm{R}_{3}<\mathrm{R}_{4} \& \mathrm{R}_{3}>\mathrm{R}_{4}$ (right)

The $\mathrm{x}$-axis refers to time while the y-axis refers to the amplitude of the signal. For the multiple-frequency signal with Gaussian White Noise, under the condition of $R_{3}=R_{4}$, the maximum extent of cancellation while place the detector close to the secondary source can reach $41.4 \%$. When $R_{1}=R_{2}$, the value arise to $48.6 \%$ under the same condition. 


\subsection{Pseudorandom binary sequence (PRBS)}
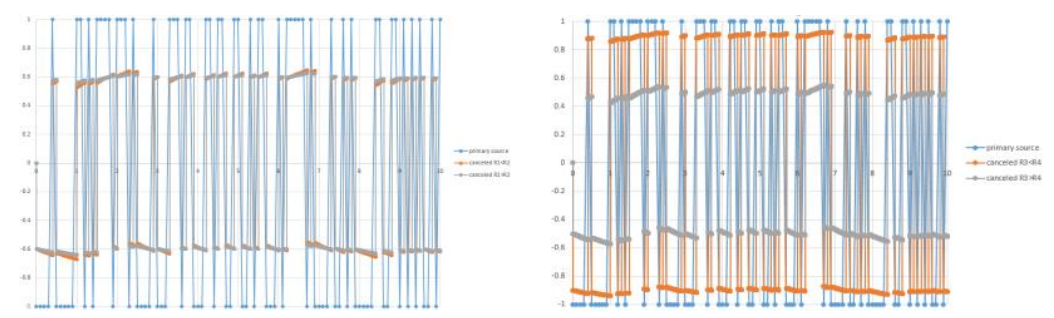

Fig. 7.1. Comparison between primary sources and canceled results: $R_{3}=R_{4}, R_{1}<$ $\mathrm{R}_{2} \& \mathrm{R}_{1}>\mathrm{R}_{2}$ (left); $\mathrm{R}_{1}=\mathrm{R}_{2}, \mathrm{R}_{3}<\mathrm{R}_{4} \& \mathrm{R}_{3}>\mathrm{R}_{4}$ (right)

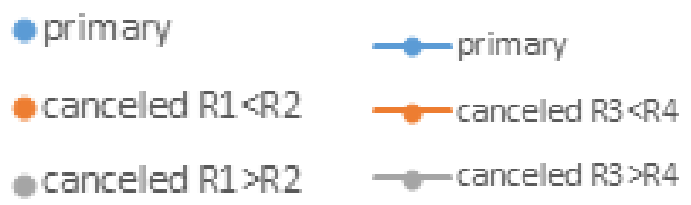

Fig. 7.2. Color explanation for simulation results: $R_{3}=R_{4}, R_{1}<R_{2} \& R_{1}>R_{2}$ (left); $R_{1}=$ $\mathrm{R}_{2}, \mathrm{R}_{3}<\mathrm{R}_{4} \& \mathrm{R}_{3}>\mathrm{R}_{4}$ (right)

The $\mathrm{x}$-axis refers to time while the $\mathrm{y}$-axis refers to the amplitude of the signal. For PRBS signal, the results are similar to the three previous tests. When $R_{3}=R_{4}$, the maximum extent of cancellation is nearly $44.3 \%$ while $R_{1}=R_{2}$, the maximum extent of cancellation can reach $60 \%$

\section{Conclusions}

Four simulated experiments have been conducted on Matlab Simulink platform. Firstly, the overall performance is good and for each different type of signal, the controller proposed by Tokhi and Leitch can achieve different extent of cancellation and it is most useful for PRBS as the maximum cancellation extent can reach $60 \%$. Secondly, according to four experiments, it is found that for a fixed controller, if the transducer placed close to the secondary source, the cancellation performance is much better. It implies that in practice, how to choose the location of the observer is crucial. Thirdly, it is important to choose the transducer to put at an equidistance from sound sources in the fixed controller as from above results, the optimal configuration is to place the observer at an equidistance from the primary source and the secondary source whilst put the observer close to the secondary source. Even good effect achieved, it still gives space for further improvement. For example, how to simplify the controller, which make it more feasible in practice and what is the optimal distance between secondary sources 
Simulated Tests of Feedforward Active Noise Control (ANC) for Building Noise Cancellation

and the observer or what is the optimal distance ratio for feedforward ANC system with a fixed controller.

\section{References}

1. Mak, C.M., Wang, Z.: Recent advances in building acoustics: an overview of prediction methods and their applications. Build. Environ 91, 118-126 (2015).

2. CHEUK, F.N.: EFFECTS OF BUILDING CONSTRUCTION NOISE ON RESIDENTS: A QUASI-EXPERIMENT. Journal of Environmental Psychology 20(4), 375-385 (2000).

3. Sang, H.P., Pyoung, J.L., Jeong, H.J.: Effects of noise sensitivity on psychophysiological responses to building noise. Build. Environ 136, 302-311 (2018).

4. Elliott, S.J., Nelson, P.A.: Active noise control. IEEE Signal Process Mag. 10(4), 12-35 (1993).

5. Leitch, R. R., Tokhi, M.O.: Active noise control systems. IEE Proceeding PS-A, 134(6), 525546 (1987)

6. George. N.V., Panda, G.: Advances in active noise control: A survey with emphasis on recent nonlinear techniques. Signal Processing 93(2), 363-377 (2013).

7. Jiang, J.J., Li, Y.: Review of active noise control techniques with emphasis on sound quality enhancement. Applied Acoustics 136, 139-148 (2018).

8. Tokhi, M.O., Wood, R.: Active Noise Control Using Multi-Layered Perceptron Neural Networks, Journal of Low Frequency Noise, Vibration and Active Control 16 (2), (1997)

9. Tokhi, M.O., Raja Ahmad, R.K.: Analysis of Geometry Related Constraints of Minimum Effort Active Noise Control System. Journal of Low Frequency Noise, Vibration and Active Control 29 (2), 111-128 (2010)

10. Tokhi, M.O., Raja Ahmad, R.K.: Theoretical Development of Minimum Effort Active Noise Control with Feedback Inclusion Architecture. Journal of Low Frequency Noise, Vibration and Active Control 27 (1), 75-81 (2008) 